\title{
molecules
}

ISSN 1420-3049

www.mdpi.com/journal/molecules

Communication

\section{TDAE Strategy for the Synthesis of 2,3-Diaryl $N$-Tosylaziridines}

\section{Omar Khoumeri, Cédric Spitz, Thierry Terme and Patrice Vanelle *}

Laboratoire de Pharmaco-Chimie Radicalaire, Faculté de Pharmacie, Institut de Chimie Radicalaire ICR, Aix-Marseille Université, UMR CNRS 7273, 27 Boulevard Jean Moulin - CS 30064 - 13385 Marseille Cedex 05, France

* Author to whom correspondence should be addressed; E-Mail: patrice.vanelle@univ-amu.fr; Tel.: +33-4-9183-5580; Fax: +33-4-9179-4677.

Received: 27 May 2013; in revised form: 14 June 2013 / Accepted: 18 June 2013 /

Published: 24 June 2013

\begin{abstract}
We report herein an original and rapid synthesis of 2,3-diaryl $N$-tosylaziridines by TDAE strategy starting from ortho- or para-nitro(dichloromethyl)benzene derivatives and $N$-tosylimines. A mixture of cis/trans isomers was isolated from 1-(dichloromethyl)-4nitrobenzene, whereas only trans-aziridines were obtained from ortho-nitro derivatives.
\end{abstract}

Keywords: TDAE; $N$-tosylimines; aziridines; diastereoselectivity

\section{Introduction}

Aziridines are found in a number of natural products exhibiting various biological properties, such as antitumor and antibiotic activities [1]. They are known to be valuable building blocks since they can undergo ring-opening reactions leading to a variety of amine products [2-5]. Therefore, the preparation of aziridines has received increasing attention in recent years. Various synthetic methods have been developed to prepare aziridines such as nitrene transfer to olefins [6-11], carbene addition to imines [12,13], aza-Darzens reaction [14], and ylide addition to imines [15,16].

Tetrakis(dimethylamino)ethylene (TDAE) is an organic reducing agent, which reacts with halogenated derivatives to generate a carbanion under mild conditions [17-19]. Since 2003, we have introduced a new program directed toward the development of original synthetic methods using TDAE methodology in medicinal chemistry [20-27]. 
In particular, we have shown that, from $o$ - and $p$-nitrobenzyl chlorides, TDAE can generate a nitrobenzyl carbanion able to react with various electrophiles such as aromatic aldehydes, $\alpha$-ketoester, ketomalonate, $\alpha$-ketolactam, and sulfonimine derivatives [28-31].

Recently, we reported the reaction of 2-(dibromomethyl)quinoxaline and 2-(dibromomethyl)-1,4dimethoxy-9,10-anthraquinone with aromatic aldehydes in the presence of TDAE, providing a mixture of cis/trans isomers of corresponding epoxides [32,33].

In order to extend this reactivity to the synthesis of aziridines, we explored the reaction of gem-dihalogenated derivatives with imines in the presence of TDAE. We chose the sulfonylaldimines for their ability to react, shown in fluorine chemistry [34] and, more recently, in anthraquinonic series [31] in the presence of TDAE. As part of our research program for new bioactive compounds [35-38], we report herein an original and efficient synthesis of 2,3-diaryl $N$-tosylaziridines using readily available $\mathrm{N}$-tosylimines and nitro(dichloromethyl)benzene derivatives by the TDAE strategy.

\section{Results and Discussion}

The required starting materials 1-3 were prepared in good yields (76-87\%) by chlorination of the corresponding aromatic benzaldehydes using $\mathrm{SOCl}_{2}$ in $\mathrm{DMF}$ at $80{ }^{\circ} \mathrm{C}$ for $2 \mathrm{~h}$ (Scheme 1). Arylsubstituted $N$-tosylimines $\mathbf{4 a}-\mathbf{g}$ were prepared by condensation of various benzaldehydes and p-toluenesulfonamide in the presence of $\mathrm{AlCl}_{3}$ in a solvent-free procedure described by Sharghi [39].

Scheme 1. Synthesis of nitro(dichloromethyl)benzene derivatives 1-3.
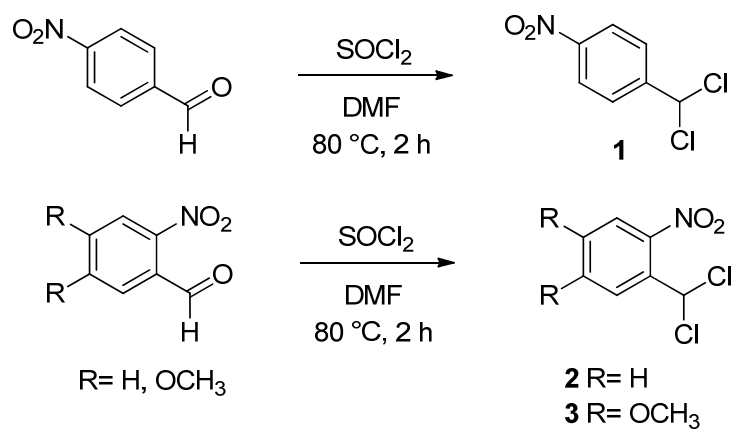

The reaction of 1-(dichloromethyl)-4-nitrobenzene $\mathbf{1}$ with two equiv. of aromatic $N$-tosylimines $\mathbf{4 a}-\mathbf{g}$ in the presence of TDAE at $-20^{\circ} \mathrm{C}$ for $1 \mathrm{~h}$, followed by $2 \mathrm{~h}$ at $\mathrm{rt}$, led to a mixture of cis/trans isomers of the corresponding aziridines $\mathbf{5 a -} \mathbf{g}$ in good yields (70-81\%) as shown in Scheme 2 and reported in Table 1. Both electron-withdrawing and electron-donating substituents on the phenyl ring of the $\mathrm{N}$-tosylimines were suitable for this reaction. ${ }^{1} \mathrm{H}-\mathrm{NMR}$ spectral studies identified the aziridines $\mathbf{5 a}-\mathbf{g}$ as trans or cis isomers by their coupling constant. Two distinct doublets appeared in 3.39-4.60 ppm region with $J=4.3-4.7 \mathrm{~Hz}$ or $J=7.3-9.4 \mathrm{~Hz}$, each of the signals corresponding to one proton. The low coupling constant here is consistent with a trans-isomer as reported in the literature [40], higher values being indicative of the cis-isomer of aziridine [41]. 
Scheme 2. TDAE-promoted reactivity of 1-(dichloromethyl)-4-nitrobenzene (1) and aromatic $N$-tosylimines $\mathbf{4 a}-\mathbf{g}$.

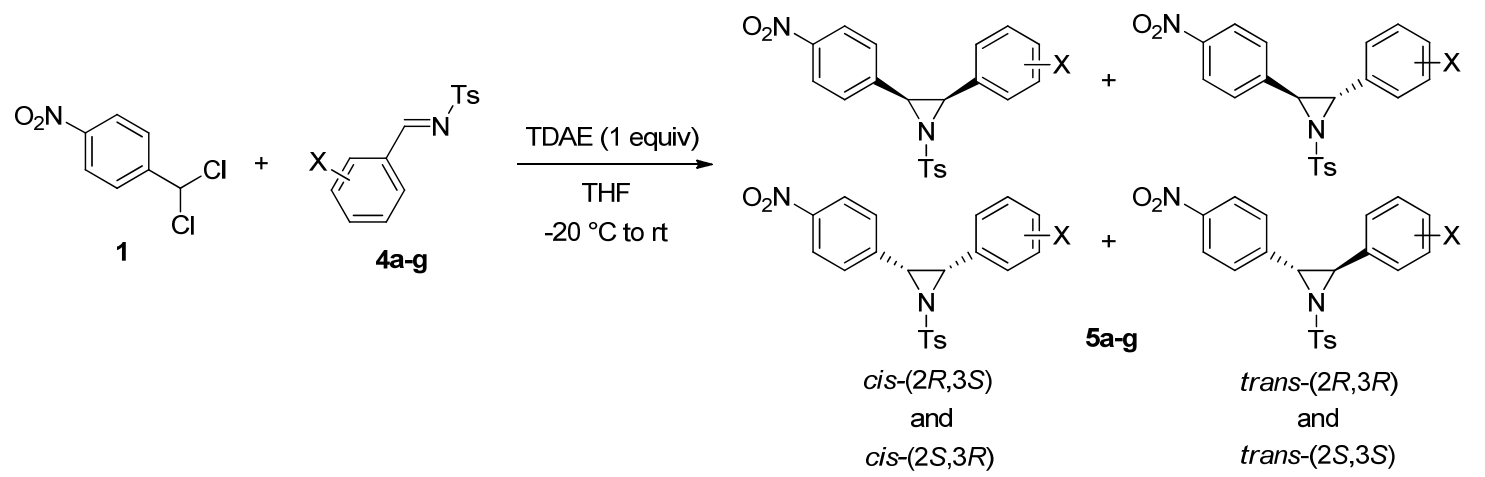

Table 1. Reaction of 1-(dichloromethyl)-4-nitrobenzene (1) with aromatic $N$-tosylimines 4a-g using TDAE strategy. ${ }^{\text {a }}$

\begin{tabular}{ccccc}
\hline Entry & $\mathbf{X}$ & Aziridine & cis/trans isomers ${ }^{\mathbf{b}} \mathbf{( \% )}$ & Yield $^{\mathbf{c}} \mathbf{( \% )}$ \\
\hline 1 & $\mathrm{H}$ & $\mathbf{5 a}$ & $86 / 14$ & 81 \\
2 & $2-\mathrm{Me}$ & $\mathbf{5 b}$ & $67 / 33$ & 74 \\
3 & $2-\mathrm{Cl}$ & $\mathbf{5 c}$ & $74 / 26$ & 70 \\
4 & $2-\mathrm{Br}$ & $\mathbf{5 d}$ & $68 / 32$ & 72 \\
5 & $3-\mathrm{F}$ & $\mathbf{5 e}$ & $86 / 14$ & 71 \\
6 & $3-\mathrm{CF}_{3}$ & $\mathbf{5 f}$ & $75 / 25$ & 73 \\
7 & $4-\mathrm{F}$ & $\mathbf{5 g}$ & $84 / 16$ & 80 \\
\hline
\end{tabular}

\footnotetext{
${ }^{a}$ All the reactions were performed using two equiv. of sulfonimines $4 \mathbf{a}-\mathbf{g}$, one equiv. of dichloride $\mathbf{1}$ and one equiv. of TDAE in anhydrous THF at $-20{ }^{\circ} \mathrm{C}$ for $1 \mathrm{~h}$ and then at $\mathrm{rt}$ for $2 \mathrm{~h} .{ }^{\mathrm{b}}$ Determined by ${ }^{1} \mathrm{H}-\mathrm{NMR}$ of the crude product. ${ }^{\mathrm{c}}$ All yields refer to chromatographically isolated pure products and are relative to dichloride $\mathbf{1}$.
}

The formation of these aziridines $\mathbf{5 a}-\mathbf{g}$ may be explained by nucleophilic addition of $\alpha$-chlorocarbanion, formed by TDAE acting with 1-(dichloromethyl)-4-nitrobenzene (1), on the $\mathrm{C}=\mathrm{N}$ double-bond of $N$-tosylimines $\mathbf{4 a}-\mathbf{g}$ followed by an intramolecular nucleophilic substitution. The greater stabilization of the cis isomer is explained by steric hindrance [15]: the largest group on the three-membered ring is the tosyl group and this will preferentially be anti to the other substituents to minimize 1,2-steric interactions, which forces the two remaining groups to be cis to each other.

The reaction of 1-(dichloromethyl)-2-nitrobenzene (2) and 1-(dichloromethyl)-4,5-dimethoxy-2nitrobenzene (3) with two equiv. of various $N$-tosylimines $4 \mathbf{a}-\mathbf{g}$ in the presence of TDAE at $-20{ }^{\circ} \mathrm{C}$ for $1 \mathrm{~h}$ followed by $2 \mathrm{~h}$ at $\mathrm{rt}$ led only to the corresponding trans-aziridines $\mathbf{6 a}-\mathbf{g}$ and $\mathbf{7 a -} \mathbf{g}$ in good yields (61-80\%) as shown in Table 2 (Scheme 3). This total trans diastereoselectivy can be explained by analysing the relevant transition states (Scheme 4). The very high steric hindrance of the ortho-nitro subtituent of $\mathbf{2}$ and $\mathbf{3}$ with aromatic ring of sulfonimines has a significant effect. Clearly, transition state $\mathbf{A}$ is less sterically hindered than transition state $\mathbf{B}$, which explains the preferential formation of the trans aziridines. To explain this total trans diastereoselectivity, a different coordination transition state could also be envisaged. In this hypothesis, the bis cation deriving from TDAE [42] coordinates both the $\mathrm{TsN}^{-}$anion and $\mathrm{NO}_{2}$ group, thus stabilizing a transition state where $\mathrm{TsN}^{-}$anion and $\mathrm{NO}_{2}$ group are on the same side like transition state $\underline{\mathbf{C}}$ and increasing the formation of the trans aziridine that must be considered the cinetic compound. 
Table 2. Reaction of 1-(dichloromethyl)-2-nitrobenzene derivatives $\mathbf{2}-\mathbf{3}$ with aromatic $N$-tosylimines $\mathbf{4 a - g}$ using TDAE strategy. ${ }^{\text {a }}$

\begin{tabular}{ccccc}
\hline Entry & Substrate & $\mathbf{X}$ & trans-Aziridine $^{\mathbf{b}}$ & Yield $^{\mathbf{c}} \mathbf{( \% )}$ \\
\hline 1 & $\mathbf{2}$ & $\mathrm{H}$ & $\mathbf{6 a}$ & 70 \\
2 & $\mathbf{2}$ & $2-\mathrm{Me}$ & $\mathbf{6 b}$ & 62 \\
3 & $\mathbf{2}$ & $2-\mathrm{Cl}$ & $\mathbf{6 c}$ & 80 \\
4 & $\mathbf{2}$ & $2-\mathrm{Br}$ & $\mathbf{6 d}$ & 70 \\
5 & $\mathbf{2}$ & $3-\mathrm{F}$ & $\mathbf{6 e}$ & 75 \\
6 & $\mathbf{2}$ & $3-\mathrm{CF}$ & $\mathbf{6 f}$ & 63 \\
7 & $\mathbf{2}$ & $4-\mathrm{F}$ & $\mathbf{6 g}$ & 79 \\
8 & $\mathbf{3}$ & $\mathrm{H}$ & $\mathbf{a}$ & 73 \\
9 & $\mathbf{3}$ & $2-\mathrm{Me}$ & $\mathbf{7 b}$ & 70 \\
10 & $\mathbf{3}$ & $2-\mathrm{Cl}$ & $\mathbf{7 d}$ & 61 \\
11 & $\mathbf{3}$ & $2-\mathrm{Br}$ & $\mathbf{7 e}$ & 74 \\
12 & $\mathbf{3}$ & $3-\mathrm{F}$ & $\mathbf{7 f}$ & 68 \\
13 & $\mathbf{3}$ & $3-\mathrm{CF}$ & $\mathbf{7 g}$ & 75 \\
14 & $\mathbf{3}$ & $4-\mathrm{F}$ & 64 \\
\hline
\end{tabular}

${ }^{a}$ All the reactions were performed using 2 equiv of sulfonimines $\mathbf{4 a}-\mathbf{g}, 1$ equiv of dichloride 2-3 and 1 equiv of TDAE in anhydrous THF at $-20{ }^{\circ} \mathrm{C}$ for $1 \mathrm{~h}$ and then at $\mathrm{rt}$ for $2 \mathrm{~h} .{ }^{\mathrm{b}}$ Determined by ${ }^{1 \mathrm{H}-\mathrm{NMR}}$ of the crude product. ${ }^{\mathrm{c}}$ All yields refer to chromatographically isolated pure products and are relative to dichloride $\mathbf{2}-\mathbf{3}$.

Scheme 3. TDAE-promoted reactivity 1-(dichloromethyl)-2-nitrobenzene derivatives 2-3 and aromatic $N$-tosylimines $\mathbf{4 a}-\mathbf{g}$.

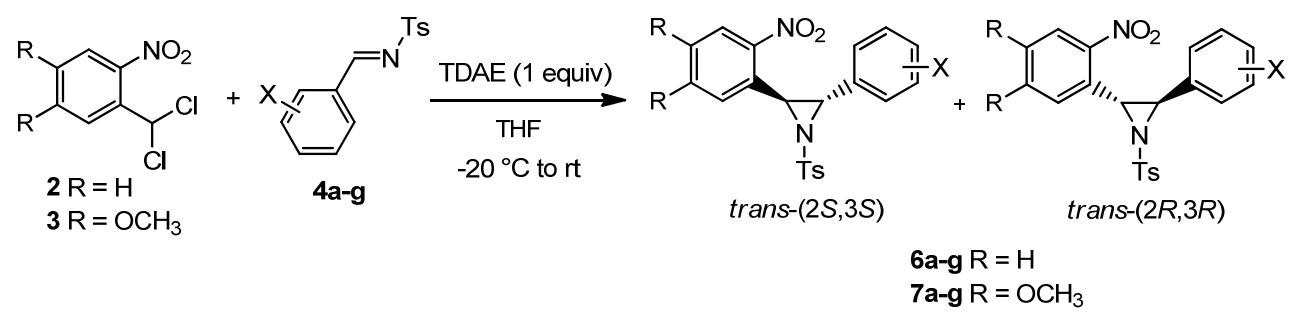

Scheme 4. Diastereoselectivity of the aziridine formation.

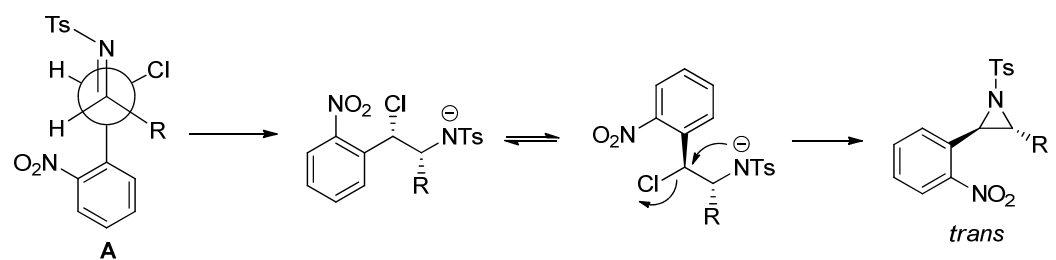<smiles>[R]C([N-]CC)[C@H](Cl)[C@H](Cl)c1ccccc1[N+](=O)[O-]</smiles>

B

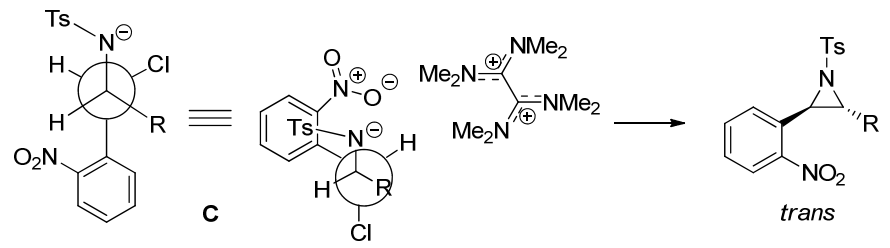




\section{Experimental}

\subsection{General}

Melting points were determined on a Büchi melting point B-540 apparatus and are uncorrected. Element analyses were performed on a Thermo Finnigan EA1112 at the spectropole of the Aix-Marseille University. Both ${ }^{1} \mathrm{H}$ - and ${ }^{13} \mathrm{C}$-NMR spectra were determined on a Bruker AC 200 spectrometer. The ${ }^{1} \mathrm{H}$ - and the ${ }^{13} \mathrm{C}$ - chemical shifts are reported from $\mathrm{CDCl}_{3}$ peaks: ${ }^{1} \mathrm{H}(7.26 \mathrm{ppm})$ and ${ }^{13} \mathrm{C}(76.9 \mathrm{ppm})$. Multiplicities are represented by the following notations: s, singlet; $\mathrm{d}$, doublet; $\mathrm{t}$, triplet; q, quartet; m, a more complex multiplet or overlapping multiplets. The following adsorbents were used for column chromatography: silica gel 60 (Merck, particle size 0.063-0.200 mm, 70-230 mesh ASTM). TLC was performed on $5 \mathrm{~cm} \times 10 \mathrm{~cm}$ aluminium plates coated with silica gel $60 \mathrm{~F}_{254}$ (Merck) in an appropriate solvent.

\subsection{General Procedure for the Preparation of $\mathbf{1}-\mathbf{3}$}

Benzaldehyde derivative $(13 \mathrm{mmol})$ was dissolved in thionyl chloride $(10 \mathrm{~mL})$, and then to the mixture was added $1 \mathrm{~mL}$ of DMF. The reaction mixture was stirred for $2 \mathrm{~h}$ at $80{ }^{\circ} \mathrm{C}$. Then, the solvent was removed under vacuum. The residue was dissolved in dichloromethane $(100 \mathrm{~mL})$, washed with $\mathrm{H}_{2} \mathrm{O}(3 \times 100 \mathrm{~mL})$ and dried over $\mathrm{MgSO}_{4}$. After evaporation, the crude product was purified by silica gel chromatography with dichloromethane: petroleum ether (1:1) to give the corresponding dichlorobenzene derivatives 1-3. Analyses for compounds $\mathbf{1}$ and $\mathbf{2}$ are in agreement with those reported in the literature $[43,44]$.

1-(Dichloromethyl)-4,5-dimethoxy-2-nitrobenzene (3). 76\% yield; white solid; mp $110{ }^{\circ} \mathrm{C}$; ${ }^{1} \mathrm{H}-\mathrm{NMR}$ $\left(200 \mathrm{MHz}, \mathrm{CDCl}_{3}\right) \delta_{\mathrm{H}} 3.98(\mathrm{~s}, 3 \mathrm{H}), 4.05(\mathrm{~s}, 3 \mathrm{H}), 7.54(\mathrm{~s}, 1 \mathrm{H}), 7.56(\mathrm{~s}, 1 \mathrm{H}), 7.73(\mathrm{~s}, 1 \mathrm{H}) ;{ }^{13} \mathrm{C}-\mathrm{NMR}$ $\left(50 \mathrm{MHz}, \mathrm{CDCl}_{3}\right) \delta_{\mathrm{C}} 56.6,56.7,66.4,107.2,110.8,129.4,149.8,153.8$. Anal. Calcd for $\mathrm{C}_{9} \mathrm{H}_{9} \mathrm{Cl}_{2} \mathrm{NO}_{4}$ : C, 40.63; H, 3.41; N, 5.26. Found: C, 40.86; H, 3.26; N, 5.39.

\subsection{General Procedure for TDAE Reaction}

Into a two-necked flask equipped with a drying tube (silica gel) and a nitrogen inlet was added $15 \mathrm{~mL}$ of an anhydrous THF solution of dichloride derivative 1-3 (1 equiv.) and $N$-tosylimine $\mathbf{4 a -} \mathbf{g}$ ( 2 equiv.). The solution was cooled to $-20{ }^{\circ} \mathrm{C}$, maintained at this temperature for $30 \mathrm{~min}$ and then was added dropwise (via a syringe) the TDAE ( 1 equiv.). The solution was vigorously stirred at $-20{ }^{\circ} \mathrm{C}$ for $1 \mathrm{~h}$ and then maintained at $\mathrm{rt}$ for $2 \mathrm{~h}$. After this time, TLC analysis $\left(\mathrm{CH}_{2} \mathrm{Cl}_{2}\right)$ clearly showed that compound (1-3) was totally consumed. The solution was filtered (to remove the octamethyl-oxamidinium dichloride) and hydrolyzed with $\mathrm{H}_{2} \mathrm{O}(70 \mathrm{~mL})$. The aqueous solution was extracted with chloroform $(3 \times 40 \mathrm{~mL})$, the combined organic layers washed with $\mathrm{H}_{2} \mathrm{O}(2 \times 40 \mathrm{~mL})$ and dried over $\mathrm{MgSO}_{4}$. Evaporation of the solvent furnished an orange viscous liquid as crude product. Purification by silica gel chromatography $\left(\mathrm{CH}_{2} \mathrm{Cl}_{2}\right.$ /petroleum ether: 70/30) and recrystallization from isopropanol gave corresponding aziridines (5-7). Analyses for compounds 5a, 5d, 5g and $6 \mathbf{6}$ are in agreement with those reported in the literature [45]. 
2-(4-Nitrophenyl)-3-o-tolyl-1-tosylaziridine (5b). cis-isomer; white solid; mp $202{ }^{\circ} \mathrm{C} ;{ }^{1} \mathrm{H}-\mathrm{NMR}(200 \mathrm{MHz}$, $\left.\mathrm{CDCl}_{3}\right) \delta_{\mathrm{H}} 2.13(\mathrm{~s}, 3 \mathrm{H}), 2.45(\mathrm{~s}, 3 \mathrm{H}), 4.28(\mathrm{~d}, 1 \mathrm{H}, J=7.3 \mathrm{~Hz}), 4.33(\mathrm{~d}, 1 \mathrm{H}, J=7.3 \mathrm{~Hz}), 6.91-7.14(\mathrm{~m}$, $4 \mathrm{H}), 7.22(\mathrm{~d}, 2 \mathrm{H}, J=8.6 \mathrm{~Hz}), 7.38(\mathrm{~d}, 2 \mathrm{H}, J=7.8 \mathrm{~Hz}), 7.87-7.99(\mathrm{~m}, 4 \mathrm{H}) .{ }^{13} \mathrm{C}-\mathrm{NMR}(50 \mathrm{MHz}$, $\left.\mathrm{CDCl}_{3}\right) \delta_{\mathrm{C}} 21.6,21.7,45.6,47.9,123.0,125.6,127.9,128.0,128.2,129.7,129.8,130.0,131.5,134.4$, 134.5, 135.9, 139.6, 145.2. trans-isomer; white solid; mp $161{ }^{\circ} \mathrm{C} ;{ }^{1} \mathrm{H}-\mathrm{NMR}\left(200 \mathrm{MHz}, \mathrm{CDCl}_{3}\right) \delta_{\mathrm{H}} 2.38$ (s, 3H), $2.41(\mathrm{~s}, 3 \mathrm{H}), 4.20(\mathrm{~d}, 1 \mathrm{H}, J=4.7 \mathrm{~Hz}), 4.35(\mathrm{~d}, 1 \mathrm{H}, J=4.7 \mathrm{~Hz}), 7.17-7.28(\mathrm{~m}, 6 \mathrm{H}), 7.59-7.66$ $(\mathrm{m}, 4 \mathrm{H}), 8.21(\mathrm{~d}, 2 \mathrm{H}, J=8.7 \mathrm{~Hz}) .{ }^{13} \mathrm{C}-\mathrm{NMR}\left(50 \mathrm{MHz}, \mathrm{CDCl}_{3}\right) \delta_{\mathrm{C}} 18.8,21.6,45.6,47.8,123.0,125.6$, 127.9, 128.0, 128.1, 128.1, 129.0, 129.7, 129.9, 134.3, 135.9, 139.5, 145.2, 147.3. Anal. Calcd for $\mathrm{C}_{22} \mathrm{H}_{20} \mathrm{~N}_{2} \mathrm{O}_{4} \mathrm{~S}: \mathrm{C}, 64.69 ; \mathrm{H}, 4.94 ; \mathrm{N}, 6.86 ; \mathrm{S}, 7.85$. Found: C, 64.79; H, 4.97; N, 6.85; S, 7.92.

2-(2-Chlorophenyl)-3-(4-nitrophenyl)-1-tosylaziridine (5c). cis-isomer; white solid; mp $193{ }^{\circ} \mathrm{C} ;{ }^{1} \mathrm{H}-\mathrm{NMR}$ $\left(200 \mathrm{MHz}, \mathrm{CDCl}_{3}\right) \delta_{\mathrm{H}} 2.45(\mathrm{~s}, 3 \mathrm{H}), 3.39(\mathrm{~d}, 1 \mathrm{H}, J=7.6 \mathrm{~Hz}), 3.46(\mathrm{~d}, 1 \mathrm{H}, J=7.6 \mathrm{~Hz}), 7.04-7.20(\mathrm{~m}$, $4 \mathrm{H}), 7.26(\mathrm{~d}, 2 \mathrm{H}, J=8.6 \mathrm{~Hz}), 7.38(\mathrm{~d}, 2 \mathrm{H}, J=8.2 \mathrm{~Hz}), 7.93$ (d, 2H, $J=8.6 \mathrm{~Hz}), 7.97$ (d, 2H, $J=8.2 \mathrm{~Hz})$. ${ }^{13} \mathrm{C}-\mathrm{NMR}\left(50 \mathrm{MHz}, \mathrm{CDCl}_{3}\right) \delta_{\mathrm{C}} 21.6,46.0,46.9,123.1,126.5,128.0,128.3,129.0,129.3,129.4,129.5$, 130.0, 133.2, 134.1, 139.1, 145.3, 147.4. trans-isomer; white solid; mp $185{ }^{\circ} \mathrm{C} ;{ }^{1} \mathrm{H}-\mathrm{NMR}(200 \mathrm{MHz}$, $\left.\mathrm{CDCl}_{3}\right) \delta_{\mathrm{H}} 2.42(\mathrm{~s}, 3 \mathrm{H}), 4.10(\mathrm{~d}, 1 \mathrm{H}, J=4.5 \mathrm{~Hz}), 4.56(\mathrm{~d}, 1 \mathrm{H}, J=4.5 \mathrm{~Hz}), 7.22-7.41(\mathrm{~m}, 6 \mathrm{H}), 7.69$ $(\mathrm{d}, 2 \mathrm{H}, J=8.7 \mathrm{~Hz}), 7.73(\mathrm{~d}, 2 \mathrm{H}, J=8.7 \mathrm{~Hz}), 8.23(\mathrm{~d}, 2 \mathrm{H}, J=8.7 \mathrm{~Hz}) .{ }^{13} \mathrm{C}-\mathrm{NMR}\left(50 \mathrm{MHz}, \mathrm{CDCl}_{3}\right)$ $\delta_{\mathrm{C}} 21.6,47.2$, 49.8, 123.5, 127.0, 127.7, 128.4, 129.4, 129.6, 130.0, 130.0, 131.2, 134.5, 136.1, 139.5, 144.8, 148.1. Anal. Calcd for $\mathrm{C}_{21} \mathrm{H}_{17} \mathrm{ClN}_{2} \mathrm{O}_{4} \mathrm{~S}: \mathrm{C}, 58.81 ; \mathrm{H}, 4.00 ; \mathrm{N}, 6.53 ; \mathrm{S}, 7.48$. Found: C, 58.88; H, $3.99 ; \mathrm{N}, 6.43 ; \mathrm{S}, 7.49$.

2-(3-Fluorophenyl)-3-(4-nitrophenyl)-1-tosylaziridine (5e). cis-isomer; white solid; mp $108{ }^{\circ} \mathrm{C}$; ${ }^{1} \mathrm{H}-\mathrm{NMR}$ $\left(200 \mathrm{MHz}, \mathrm{CDCl}_{3}\right) \delta_{\mathrm{H}} 2.47(\mathrm{~s}, 3 \mathrm{H}), 4.22(\mathrm{~d}, 1 \mathrm{H}, J=9.4 \mathrm{~Hz}), 4.32(\mathrm{~d}, 1 \mathrm{H}, J=9.4 \mathrm{~Hz}), 6.69-6.88(\mathrm{~m}$, $3 \mathrm{H}), 7.04-7.16(\mathrm{~m}, 1 \mathrm{H}), 7.23(\mathrm{~d}, 2 \mathrm{H}, J=8.3 \mathrm{~Hz}), 7.39$ (d, 2H, $J=8.3 \mathrm{~Hz}), 7.96(\mathrm{~d}, 2 \mathrm{H}, J=8.3 \mathrm{~Hz})$ $7.99(\mathrm{~d}, 2 \mathrm{H}, J=8.3 \mathrm{~Hz}) .{ }^{13} \mathrm{C}-\mathrm{NMR}\left(50 \mathrm{MHz}, \mathrm{CDCl}_{3}\right) \delta_{\mathrm{C}} 21.7,46.3,47.1(\mathrm{~d}, J=2.6 \mathrm{~Hz}), 114.5(\mathrm{~d}$, $J=22.7 \mathrm{~Hz}), 115.2(\mathrm{~d}, J=21.1 \mathrm{~Hz}) 123.2(\mathrm{~d}, J=2.9 \mathrm{~Hz}), 123.3,128.0,128.5,129.9,130.1,133.7$ (d, $J=8.0 \mathrm{~Hz}), 134.2,139.1,145.4,147.6,162.4(\mathrm{~d}, J=247.0 \mathrm{~Hz})$. trans-isomer; white solid; $\mathrm{mp} 143{ }^{\circ} \mathrm{C}$; ${ }^{1} \mathrm{H}-\mathrm{NMR}\left(200 \mathrm{MHz}, \mathrm{CDCl}_{3}\right) \delta_{\mathrm{H}} 2.41(\mathrm{~s}, 3 \mathrm{H}), 4.22(\mathrm{~d}, 1 \mathrm{H}, J=4.4 \mathrm{~Hz}), 4.26(\mathrm{~d}, 1 \mathrm{H}, J=4.4 \mathrm{~Hz}), 7.70$ $(\mathrm{d}, 2 \mathrm{H}, J=8.8 \mathrm{~Hz}), 7.24-7.41(\mathrm{~m}, 4 \mathrm{H}), 7.60(\mathrm{~d}, 2 \mathrm{H}, J=8.8 \mathrm{~Hz}), 7.67(\mathrm{~d}, 2 \mathrm{H}, J=8.2 \mathrm{~Hz}), 8.21(\mathrm{~d}, 2 \mathrm{H}$, $J=8.8 \mathrm{~Hz}) .{ }^{13} \mathrm{C}$ NMR $\left(50 \mathrm{MHz}, \mathrm{CDCl}_{3}\right) \delta_{\mathrm{C}} 21.6,49.0,50.1(\mathrm{~d}, J=2.2 \mathrm{~Hz}), 115.2(\mathrm{~d}, J=22.7 \mathrm{~Hz})$, $116.1(\mathrm{~d}, J=21.2 \mathrm{~Hz}), 123.7,124.0(\mathrm{~d}, J=2.9 \mathrm{~Hz}), 127.5,129.2,129.7,130.0,134.7(\mathrm{~d}, J=7.7 \mathrm{~Hz})$, 136.4, 140.2, 144.7, 148.1, 162.7 (d, $J=247.4 \mathrm{~Hz}$ ). Anal. Calcd for $\mathrm{C}_{21} \mathrm{H}_{17} \mathrm{FN}_{2} \mathrm{O}_{4} \mathrm{~S}$ : C, 61.16; H, 4.15; N, 6.79; S, 7.77. Found: C, 60.51; H, 4.19; N, 6.62; S, 7.66.

2-(4-Nitrophenyl)-3-(3-(trifluoromethyl)phenyl)-1-tosyl-aziridine (5f). cis-isomer; white solid; mp $63{ }^{\circ} \mathrm{C}$; ${ }^{1} \mathrm{H}-\mathrm{NMR}\left(200 \mathrm{MHz}, \mathrm{CDCl}_{3}\right) \delta_{\mathrm{H}} 2.44(\mathrm{~s}, 3 \mathrm{H}), 4.30(\mathrm{~d}, 1 \mathrm{H}, J=7.7 \mathrm{~Hz}), 4.34$ (d, 1H, $\left.J=7.7 \mathrm{~Hz}\right), 7.21-7.41$ $(\mathrm{m}, 8 \mathrm{H}), 7.95(\mathrm{~d}, 2 \mathrm{H}, J=8.4 \mathrm{~Hz}), 7.99(\mathrm{~d}, 2 \mathrm{H}, J=8.4 \mathrm{~Hz}) .{ }^{13} \mathrm{C}-\mathrm{NMR}\left(50 \mathrm{MHz}, \mathrm{CDCl}_{3}\right) \delta_{\mathrm{C}} 21.6,46.4$, $46.9,123.3,124.4$ (q, $J=4.0 \mathrm{~Hz}), 125.0$ (q, $J=4.0 \mathrm{~Hz}), 128.0,128.5,128.8,130.0,130.5$ (q, $J=33.0 \mathrm{~Hz})$, 130.7, 132.3, 133.9, 138.9, $142.5(\mathrm{q}, J=238.9 \mathrm{~Hz}), 145.6,147.5$. trans-isomer; white solid; $\mathrm{mp} 164{ }^{\circ} \mathrm{C}$; ${ }^{1} \mathrm{H}-\mathrm{NMR}\left(200 \mathrm{MHz}, \mathrm{CDCl}_{3}\right) \delta_{\mathrm{H}} 2.40(\mathrm{~s}, 3 \mathrm{H}), 4.25(\mathrm{~d}, 1 \mathrm{H}, J=4.3 \mathrm{~Hz}), 4.35(\mathrm{~d}, 1 \mathrm{H}, J=4.3 \mathrm{~Hz})$, 7.20-7.25 (m, 4H), 7.54-7.65 (m, 4H), $7.97(\mathrm{~d}, 2 \mathrm{H}, J=8.8 \mathrm{~Hz}), 8.20(\mathrm{~d}, 2 \mathrm{H}, J=8.8 \mathrm{~Hz}) .{ }^{13} \mathrm{C}-\mathrm{NMR}$ $\left(50 \mathrm{MHz}, \mathrm{CDCl}_{3}\right) \delta_{\mathrm{C}} 21.5,48.5,50.1,123.7,125.3(\mathrm{q}, J=3.7 \mathrm{~Hz}), 125.8(\mathrm{q}, J=3.7 \mathrm{~Hz}), 127.5,129.1$, 
129.7, 130.1, 130.9 (q, $J=32.6 \mathrm{~Hz}), 131.6,133.1,136.1,140.1,140.5$ (q, $J=238.1 \mathrm{~Hz}), 144.9,148.1$. Anal. Calcd for $\mathrm{C}_{22} \mathrm{H}_{17} \mathrm{~F}_{3} \mathrm{~N}_{2} \mathrm{O}_{4} \mathrm{~S}$ : C, 57.14; H, 3.71; N, 6.06; S, 6.93. Found: C, 55.46; H, 3.74; N, 5.92; S, 6.71 .

trans-2-(2-Nitrophenyl)-3-o-tolyl-1-tosylaziridine (6b). White solid; mp $160{ }^{\circ} \mathrm{C} ;{ }^{1} \mathrm{H}-\mathrm{NMR}(200 \mathrm{MHz}$, $\left.\mathrm{CDCl}_{3}\right) \delta_{\mathrm{H}} 2.27(\mathrm{~s}, 3 \mathrm{H}), 2.41(\mathrm{~s}, 3 \mathrm{H}), 3.87(\mathrm{~d}, 1 \mathrm{H}, J=4.8 \mathrm{~Hz}), 5.16(\mathrm{~d}, 1 \mathrm{H}, J=4.8 \mathrm{~Hz}), 7.16-7.20(\mathrm{~m}$, 4H), 7.26-7.32 (m, 1H), 7.48-7.77 (m, 6H), $8.15(\mathrm{dd}, 1 \mathrm{H}, J=8.1,1.1 \mathrm{~Hz}) .{ }^{13} \mathrm{C}-\mathrm{NMR}(50 \mathrm{MHz}$, $\left.\mathrm{CDCl}_{3}\right) \delta_{\mathrm{C}} 19.3,21.5,43.6,51.9,124.9,125.7,127.9,128.5,128.6,129.1,129.2,129.4,129.7,129.8$, 131.4, 134.2, 135.4, 139.6, 144.3, 148.1. Anal. Calcd for $\mathrm{C}_{22} \mathrm{H}_{20} \mathrm{~N}_{2} \mathrm{O}_{4} \mathrm{~S}: \mathrm{C}, 64.69 ; \mathrm{H}, 4.94 ; \mathrm{N}, 6.86$; S, 7.85. Found: C, 64.81; H, 4.96; N, 6.82; S, 7.57.

trans-2-(2-Chlorophenyl)-3-(2-nitrophenyl)-1-tosyl-aziridine (6c). White solid; mp $153{ }^{\circ} \mathrm{C} ;{ }^{1} \mathrm{H}-\mathrm{NMR}$ $\left(200 \mathrm{MHz}, \mathrm{CDCl}_{3}\right) \delta_{\mathrm{H}} 2.42(\mathrm{~s}, 3 \mathrm{H}), 4.25(\mathrm{~d}, 1 \mathrm{H}, J=4.8 \mathrm{~Hz}), 5.04(\mathrm{~d}, 1 \mathrm{H}, J=4.8 \mathrm{~Hz}), 7.21-7.35(\mathrm{~m}$, $5 \mathrm{H}), 7.51-7.72(\mathrm{~m}, 5 \mathrm{H}), 7.87(\mathrm{~d}, 1 \mathrm{H}, J=7.6 \mathrm{~Hz}), 8.20(\mathrm{~d}, 1 \mathrm{H}, J=7.6 \mathrm{~Hz}) .{ }^{13} \mathrm{C}-\mathrm{NMR}(50 \mathrm{MHz}$, $\left.\mathrm{CDCl}_{3}\right) \delta_{\mathrm{C}} 21.6,46.0,49.3,125.0,126.4,127.8,129.1,129.5,129.6,129.9,130.0,130.1,130.2,130.3$, 134.2, 135.7, 136.0, 144.6, 148.5. Anal. Calcd for $\mathrm{C}_{21} \mathrm{H}_{17} \mathrm{ClN}_{2} \mathrm{O}_{4} \mathrm{~S}: \mathrm{C}, 58.81 ; \mathrm{H}, 4.00 ; \mathrm{N}, 6.53 ; \mathrm{S}, 7.48$. Found: C, 58.72; H, 3.99; N, 6.50; S, 7.46.

trans-2-(2-Bromophenyl)-3-(2-nitrophenyl)-1-tosyl-aziridine (6d). White solid; mp $153{ }^{\circ} \mathrm{C}$; ${ }^{1} \mathrm{H}-\mathrm{NMR}$ $\left(200 \mathrm{MHz}, \mathrm{CDCl}_{3}\right) \delta_{\mathrm{H}} 2.41(\mathrm{~s}, 3 \mathrm{H}), 4.26(\mathrm{~d}, 1 \mathrm{H}, J=4.9 \mathrm{~Hz}), 5.00(\mathrm{~d}, 1 \mathrm{H}, J=4.9 \mathrm{~Hz}), 7.21-7.26(\mathrm{~m}$, 2H), 7.29-7.42 (m, 2H), 7.51-7.72 (m, 6H), 7.89-7.92 (m, 1H), 8.18 (dd, 1H, $J=8.1 \mathrm{~Hz}, J=1.0 \mathrm{~Hz})$. ${ }^{13} \mathrm{C}-\mathrm{NMR}\left(50 \mathrm{MHz}, \mathrm{CDCl}_{3}\right) \delta_{\mathrm{C}} 21.5,46.4,51.1,125.0,127.3,127.8,128.1,129.4,129.6,129.7,129.8$, 130.0, 130.3, 131.3, 132.3, 134.1, 135.6, 144.6, 148.5. Anal. Calcd for $\mathrm{C}_{21} \mathrm{H}_{17} \mathrm{BrN}_{2} \mathrm{O}_{4} \mathrm{~S}: \mathrm{C}, 53.29 ; \mathrm{H}$, 3.62; N, 5.92; S, 6.77. Found: C, 53.36; H, 3.66; N, 5.96; S, 6.78.

trans-2-(3-Fluorophenyl)-3-(2-nitrophenyl)-1-tosyl-aziridine (6e). White solid; mp $154{ }^{\circ} \mathrm{C}$; ${ }^{1} \mathrm{H}-\mathrm{NMR}$ $\left(200 \mathrm{MHz}, \mathrm{CDCl}_{3}\right) \delta_{\mathrm{H}} 2.42(\mathrm{~s}, 3 \mathrm{H}), 3.91(\mathrm{~d}, 1 \mathrm{H}, J=4.6 \mathrm{~Hz}), 5.03(\mathrm{~d}, 1 \mathrm{H}, J=4.6 \mathrm{~Hz}), 7.01-7.26(\mathrm{~m}$, 4H), 7.31-7.36 (m, 2H), 7.48-7.69 (m, 5H), $8.17(\mathrm{~d}, 1 \mathrm{H}, J=7.9 \mathrm{~Hz}) .{ }^{13} \mathrm{C}-\mathrm{NMR}\left(50 \mathrm{MHz}, \mathrm{CDCl}_{3}\right) \delta_{\mathrm{C}}$ 21.6, 45.5, $51.6(\mathrm{~d}, J=2.2 \mathrm{~Hz}), 116.1(\mathrm{~d}, J=20.8 \mathrm{~Hz}), 116.5(\mathrm{~d}, J=22.7 \mathrm{~Hz}), 125.0,125.3(\mathrm{~d}, J=2.9 \mathrm{~Hz})$, 127.8, 129.4, 129.5, 129.8, 129.9, 130.5, 133.1 (d, $J=8.0 \mathrm{~Hz}), 134.2,135.8,144.6,148.2,162.4(\mathrm{~d}$, $J=246.6 \mathrm{~Hz}$ ). Anal. Calcd for $\mathrm{C}_{21} \mathrm{H}_{17} \mathrm{FN}_{2} \mathrm{O}_{4} \mathrm{~S}: \mathrm{C}, 61.16$; H, 4.15; N, 6.79; S, 7.77. Found: C, 61.29; H, $4.20 ; \mathrm{N}, 6.75 ; \mathrm{S}, 7.72$.

trans-2-(2-Nitrophenyl)-3-(3-(trifluoromethyl)phenyl)-aziridine (6f). White solid; mp $145{ }^{\circ} \mathrm{C} ;{ }^{1} \mathrm{H}-\mathrm{NMR}$ $\left(200 \mathrm{MHz}, \mathrm{CDCl}_{3}\right) \delta_{\mathrm{H}} 2.41(\mathrm{~s}, 3 \mathrm{H}), 3.91(\mathrm{~d}, 1 \mathrm{H}, J=4.5 \mathrm{~Hz}), 5.13(\mathrm{~d}, 1 \mathrm{H}, J=4.5 \mathrm{~Hz}), 7.21(\mathrm{~d}, 2 \mathrm{H}$, $J=8.1 \mathrm{~Hz}), 7.49-7.84(\mathrm{~m}, 9 \mathrm{H}), 8.18(\mathrm{~d}, 1 \mathrm{H}, J=8.4 \mathrm{~Hz}) .{ }^{13} \mathrm{C}-\mathrm{NMR}\left(50 \mathrm{MHz}, \mathrm{CDCl}_{3}\right) \delta_{\mathrm{C}} 21.5,44.9$, 51.6, 121.5 (q, $J=272.2 \mathrm{~Hz}), 125.0,125.8$ (q, $J=3.7 \mathrm{~Hz}), 126.8$ (q, $J=3.7 \mathrm{~Hz}), 127.7,128.9,129.5$, 129.6, 129.8, 130.4 (q, $J=32.2 \mathrm{~Hz}), 130.6,131.5,132.8,134.3,135.5,144.8,148.1$. Anal. Calcd for $\mathrm{C}_{22} \mathrm{H}_{17} \mathrm{~F}_{3} \mathrm{~N}_{2} \mathrm{O}_{4} \mathrm{~S}$ : C, 57.14; H, 3.71; N, 6.06; S, 6.93. Found: C, 56.96; H, 3.72; N, 6.11; S, 6.72.

trans-2-(4-Fluorophenyl)-3-(2-nitrophenyl)-1-tosylaziridine (6g). White solid; mp $135{ }^{\circ} \mathrm{C}$; ${ }^{1} \mathrm{H}-\mathrm{NMR}$ $\left(200 \mathrm{MHz}, \mathrm{CDCl}_{3}\right) \delta_{\mathrm{H}} 2.42(\mathrm{~s}, 3 \mathrm{H}), 3.86(\mathrm{~d}, 1 \mathrm{H}, J=4.6 \mathrm{~Hz}), 5.10(\mathrm{~d}, 1 \mathrm{H}, J=4.6 \mathrm{~Hz}), 7.04(\mathrm{t}, 2 \mathrm{H}$, $J=8.4 \mathrm{~Hz}), 7.23(\mathrm{t}, 2 \mathrm{H}, J=8.4 \mathrm{~Hz}), 7.47-7.63(\mathrm{~m}, 7 \mathrm{H}), 8.15(\mathrm{~d}, 1 \mathrm{H}, J=7.8 \mathrm{~Hz}) .{ }^{13} \mathrm{C}-\mathrm{NMR}(50 \mathrm{MHz}$, 
$\left.\mathrm{CDCl}_{3}\right) \delta_{\mathrm{C}} 21.6,44.9,52.4,115.3(\mathrm{~d}, J=21.6 \mathrm{~Hz}), 125.0,126.2(\mathrm{~d}, J=3.3 \mathrm{~Hz}), 127.7,129.3,129.5$, 129.6, 131.1, $131.7(\mathrm{~d}, J=8.4 \mathrm{~Hz}), 134.3,136.0,144.5,148.1,162.5$ (d, $J=248.4 \mathrm{~Hz})$. Anal. Calcd for $\mathrm{C}_{21} \mathrm{H}_{17} \mathrm{FN}_{2} \mathrm{O}_{4} \mathrm{~S}: \mathrm{C}, 61.16 ; \mathrm{H}, 4.15 ; \mathrm{N}, 6.79 ; \mathrm{S}, 7.77$. Found: C, 61.31; H, 4.20; N, 6.79; S, 7.71.

trans-2-(4,5-Dimethoxy-2-nitrophenyl)-3-phenyl-1-tosylaziridine (7a). White solid; mp $154{ }^{\circ} \mathrm{C}$; ${ }^{1} \mathrm{H}-\mathrm{NMR}$ $\left(200 \mathrm{MHz}, \mathrm{CDCl}_{3}\right) \delta_{\mathrm{H}} 2.39(\mathrm{~s}, 3 \mathrm{H}), 3.74(\mathrm{~s}, 3 \mathrm{H}), 3.88(\mathrm{~d}, 1 \mathrm{H}, J=4.4 \mathrm{~Hz}), 3.94(\mathrm{~s}, 3 \mathrm{H}), 5.15(\mathrm{~d}, 1 \mathrm{H}$, $J=4.4 \mathrm{~Hz}), 6.91(\mathrm{~s}, 1 \mathrm{H}), 7.19-7.23(\mathrm{~m}, 2 \mathrm{H}), 7.34-7.37(\mathrm{~m}, 3 \mathrm{H}), 7.59-7.63(\mathrm{~m}, 4 \mathrm{H}), 7.71(\mathrm{~s}, 1 \mathrm{H})$. ${ }^{13} \mathrm{C}-\mathrm{NMR}\left(50 \mathrm{MHz}, \mathrm{CDCl}_{3}\right) \delta_{\mathrm{C}} 21.5,45.3,53.7,56.1,56.4,107.8,110.6,126.1,127.8,128.2,129.0$, $129.5,129.9,130.2,136.5,140.3,144.2,148.5,153.7$. Anal. Calcd for $\mathrm{C}_{23} \mathrm{H}_{22} \mathrm{~N}_{2} \mathrm{O}_{6} \mathrm{~S}: \mathrm{C}, 60.78 ; \mathrm{H}$, $4.88 ; \mathrm{N}, 6.16 ; \mathrm{S}, 7.06$. Found: C, 60.80; H, 4.92; N, 6.20; S, 7.03.

trans-2-(4,5-Dimethoxy-2-nitrophenyl)-3-o-tolyl-1-tosylaziridine (7b). White solid; mp $167{ }^{\circ} \mathrm{C} ;{ }^{1} \mathrm{H}-\mathrm{NMR}$ $\left(200 \mathrm{MHz}, \mathrm{CDCl}_{3}\right) \delta_{\mathrm{H}} 2.38(\mathrm{~s}, 6 \mathrm{H}), 3.76(\mathrm{~s}, 3 \mathrm{H}), 3.82(\mathrm{~d}, 1 \mathrm{H}, J=4.8 \mathrm{~Hz}), 3.93(\mathrm{~s}, 3 \mathrm{H}), 5.18(\mathrm{~d}, 1 \mathrm{H}$, $J=4.8 \mathrm{~Hz}), 6.98(\mathrm{~s}, 1 \mathrm{H}), 7.18(\mathrm{~d}, 4 \mathrm{H}, J=7.3 \mathrm{~Hz}), 7.24-7.32(\mathrm{~m}, 2 \mathrm{H}), 7.56(\mathrm{~d}, 1 \mathrm{H}, J=8.2 \mathrm{~Hz}), 7.65$ $(\mathrm{d}, 1 \mathrm{H}, J=7.3 \mathrm{~Hz}), 7.70(\mathrm{~s}, 1 \mathrm{H}) .{ }^{13} \mathrm{C}-\mathrm{NMR}\left(50 \mathrm{MHz}, \mathrm{CDCl}_{3}\right) \delta_{\mathrm{C}} 19.4,21.4,44.3,52.5,56.1,56.4$, $107.8,110.7,125.7,126.4,127.9,128.5,128.7,129.2,129.4,129.8,135.9,139.9,140.2,144.2,148.4$, 153.7. Anal. Calcd for $\mathrm{C}_{24} \mathrm{H}_{24} \mathrm{~N}_{2} \mathrm{O}_{6} \mathrm{~S}: \mathrm{C}, 61.52 ; \mathrm{H}, 5.16 ; \mathrm{N}, 5.98 ; \mathrm{S}, 6.84$. Found: $\mathrm{C}, 61.86 ; \mathrm{H}, 5.21 ; \mathrm{N}$, $5.98 ; \mathrm{S}, 6.78$.

trans-2-(2-Chlorophenyl)-3-(4,5-dimethoxy-2-nitrophenyl)-1-tosylaziridine (7c). White solid; mp $144{ }^{\circ} \mathrm{C}$; ${ }^{1} \mathrm{H}-\mathrm{NMR}\left(200 \mathrm{MHz}, \mathrm{CDCl}_{3}\right) \delta_{\mathrm{H}} 2.41(\mathrm{~s}, 3 \mathrm{H}), 3.83(\mathrm{~s}, 3 \mathrm{H}), 3.95(\mathrm{~s}, 3 \mathrm{H}), 4.17(\mathrm{~d}, 1 \mathrm{H}, J=4.9 \mathrm{~Hz}), 5.07$ $(\mathrm{d}, 1 \mathrm{H}, J=4.9 \mathrm{~Hz}), 7.09(\mathrm{~s}, 1 \mathrm{H}), 7.24(\mathrm{~d}, 2 \mathrm{H}, J=8.2 \mathrm{~Hz}), 7.29-7.40(\mathrm{~m}, 3 \mathrm{H}), 7.65(\mathrm{~d}, 2 \mathrm{H}, J=8.2 \mathrm{~Hz})$, 7.72-7.76 (m, 2H). ${ }^{13} \mathrm{C}-\mathrm{NMR}\left(50 \mathrm{MHz}, \mathrm{CDCl}_{3}\right) \delta_{\mathrm{C}} 21.5,46.3,50.0,56.2,56.4,107.9,111.3,125.0$, 126.8, 127.9, 129.1, 129.4, 129.5, 130.1, 130.3, 136.0, 136.4, 140.8, 144.5, 148.8, 153.6. Anal. Calcd for $\mathrm{C}_{23} \mathrm{H}_{21} \mathrm{ClN}_{2} \mathrm{O}_{6} \mathrm{~S}: \mathrm{C}, 56.50 ; \mathrm{H}, 4.33 ; \mathrm{N}, 5.73 ; \mathrm{S}, 6.56$. Found: $\mathrm{C}, 56.44 ; \mathrm{H}, 4.33 ; \mathrm{N}, 5.71 ; \mathrm{S}, 6.57$.

trans-2-(2-Bromophenyl)-3-(4,5-dimethoxy-2-nitrophenyl)-1-tosylaziridine (7d). White solid; mp $164{ }^{\circ} \mathrm{C}$; ${ }^{1} \mathrm{H}-\mathrm{NMR}\left(200 \mathrm{MHz} \mathrm{CDCl}_{3}\right) \delta_{\mathrm{H}} 2.40(\mathrm{~s}, 3 \mathrm{H}), 3.85(\mathrm{~s}, 3 \mathrm{H}), 3.94(\mathrm{~s}, 3 \mathrm{H}), 4.19(\mathrm{~d}, 1 \mathrm{H}, J=4.8 \mathrm{~Hz}), 5.02$ (d, 1H, $J=4.8 \mathrm{~Hz}), 7.14(\mathrm{~s}, 1 \mathrm{H}), 7.21-7.25(\mathrm{~m}, 3 \mathrm{H}), 7.34$ (t, 2H, J=7.3 Hz), 7.53-7.72 (m, 3H), 7.74 (s, 1H). ${ }^{13} \mathrm{C}-\mathrm{NMR}\left(50 \mathrm{MHz}, \mathrm{CDCl}_{3}\right) \delta_{\mathrm{C}} 21.5,47.0,51.7,56.3,56.4,107.9,111.6,124.6,126.3,127.4$, $127.9,129.5,130.1,130.4,131.2,132.3,135.9,140.9,144.5,148.8,153.4$. Anal. Calcd for $\mathrm{C}_{23} \mathrm{H}_{21} \mathrm{BrN}_{2} \mathrm{O}_{6} \mathrm{~S}$ : C, 51.79; H, 3.97; N, 5.25; S, 6.01. Found: C, 51.77; H, 3.93; N, 5.22; S, 5.88.

trans-2-(4,5-Dimethoxy-2-nitrophenyl)-3-(3-fluorophenyl)-1-tosylaziridine (7e). White solid; mp $161{ }^{\circ} \mathrm{C}$; ${ }^{1} \mathrm{H}-\mathrm{NMR}\left(200 \mathrm{MHz}, \mathrm{CDCl}_{3}\right) \delta_{\mathrm{H}} 2.41(\mathrm{~s}, 3 \mathrm{H}), 3.75(\mathrm{~s}, 3 \mathrm{H}), 3.84(\mathrm{~d}, 1 \mathrm{H}, J=4.4 \mathrm{~Hz}), 3.95(\mathrm{~s}, 3 \mathrm{H}), 5.08$ $(\mathrm{d}, 1 \mathrm{H}, J=4.4 \mathrm{~Hz}), 6.91(\mathrm{~s}, 1 \mathrm{H}), 7.03-7.12(\mathrm{~m}, 1 \mathrm{H}), 7.23-7.29(\mathrm{~m}, 3 \mathrm{H}), 7.33-7.45(\mathrm{~m}, 2 \mathrm{H}), 7.65(\mathrm{~d}$, $2 \mathrm{H}, J=8.3 \mathrm{~Hz}), 7.72(\mathrm{~s}, 1 \mathrm{H}) .{ }^{13} \mathrm{C}-\mathrm{NMR}\left(50 \mathrm{MHz}, \mathrm{CDCl}_{3}\right) \delta_{\mathrm{C}} 21.6,45.7,52.7,56.2,56.5,107.9,110.7$, $116.2(\mathrm{~d}, J=20.8 \mathrm{~Hz}), 116.9(\mathrm{~d}, J=22.7 \mathrm{~Hz}), 125.7,127.9,129.5,129.6,129.8$ (d, $J=8.0 \mathrm{~Hz}), 132.9$ $(\mathrm{d}, J=8.0 \mathrm{~Hz}), 136.3,144.6,148.7,153.8,160.0,162.5(\mathrm{~d}, J=248.6 \mathrm{~Hz})$. Anal. Calcd for $\mathrm{C}_{23} \mathrm{H}_{21} \mathrm{FN}_{2} \mathrm{O}_{6} \mathrm{~S}: \mathrm{C}, 58.47 ; \mathrm{H}, 4.48 ; \mathrm{N}, 5.93 ; \mathrm{S}, 6.79$. Found: C, 58.55; H, 4.54; N, 5.92; S, 6.76.

trans-2-(4,5-Dimethoxy-2-nitrophenyl)-3-(3-(trifluoromethyl)phenyl)-1-tosylaziridine (7f). White solid; mp $163{ }^{\circ} \mathrm{C} ;{ }^{1} \mathrm{H}-\mathrm{NMR}\left(200 \mathrm{MHz}, \mathrm{CDCl}_{3}\right) \delta_{\mathrm{H}} 2.39$ (s, 3H), $2.76(\mathrm{~s}, 3 \mathrm{H}), 3.85$ (d, 1H, J = 4.5 Hz), 3.93 
(s, 3H), $5.14(\mathrm{~d}, 1 \mathrm{H}, J=4.5 \mathrm{~Hz}), 6.94(\mathrm{~s}, 1 \mathrm{H}), 7.21(\mathrm{~d}, 2 \mathrm{H}, J=8.1 \mathrm{~Hz}), 7.48-7.68(\mathrm{~m}, 5 \mathrm{H}), 7.71$ $(\mathrm{s}, 1 \mathrm{H}), 7.88(\mathrm{~d}, 1 \mathrm{H}, J=7.4 \mathrm{~Hz}) .{ }^{13} \mathrm{C}-\mathrm{NMR}\left(50 \mathrm{MHz}, \mathrm{CDCl}_{3}\right) \delta_{\mathrm{C}} 21.4,45.3,52.3,56.2,56.4,107.8$, 110.6, 125.5 (q, $J=272.6 \mathrm{~Hz}), 125.8$ (q, $J=3.7 \mathrm{~Hz}), 126.9$ (q, $J=4.0 \mathrm{~Hz}), 127.7,128.8,128.8,129.6$, $130.4(\mathrm{q}, J=32.6 \mathrm{~Hz}), 131.3,133.1,135.9,140.2,144.7,148.7,153.8$. Anal. Calcd for $\mathrm{C}_{24} \mathrm{H}_{21} \mathrm{~F}_{3} \mathrm{~N}_{2} \mathrm{O}_{6} \mathrm{~S}: \mathrm{C}, 55.17 ; \mathrm{H}, 4.05 ; \mathrm{N}, 5.36 ; \mathrm{S}, 6.14$. Found: C, 55.21; H, 4.19; N, 5.41; S, 6.05 .

trans-2-(4,5-Dimethoxy-2-nitrophenyl)-3-(4-fluorophenyl)-1-tosylaziridine (7g). White solid; mp $158{ }^{\circ} \mathrm{C}$; ${ }^{1} \mathrm{H}-\mathrm{NMR}\left(200 \mathrm{MHz}, \mathrm{CDCl}_{3}\right) \delta_{\mathrm{H}} 2.41(\mathrm{~s}, 3 \mathrm{H}), 3.72(\mathrm{~s}, 3 \mathrm{H}), 3.82(\mathrm{~d}, 1 \mathrm{H}, J=4.4 \mathrm{~Hz}), 3.90$ (s, 3H), 5.13 $(\mathrm{d}, 1 \mathrm{H}, J=4.4 \mathrm{~Hz}), 6.86(\mathrm{~s}, 1 \mathrm{H}), 7.06(\mathrm{t}, 2 \mathrm{H}, J=8.6 \mathrm{~Hz}), 7.23-7.28(\mathrm{~m}, 2 \mathrm{H}), 7.58-7.78(\mathrm{~m}, 5 \mathrm{H}) .{ }^{13} \mathrm{C}-\mathrm{NMR}$ $\left(50 \mathrm{MHz}, \mathrm{CDCl}_{3}\right) \delta_{\mathrm{C}} 21.5,45.5,53.1,56.1,56.4,107.9,110.5,115.3(\mathrm{~d}, J=21.6 \mathrm{~Hz}), 126.1(\mathrm{~d}, J=2.2$ Hz), 126.2, 127.8, 129.6, 131.9 (d, $J=8.4 \mathrm{~Hz}), 136.6,140.3,144.4,148.6,153.8,162.8$ (d, $J=248.8$ Hz). Anal. Calcd for $\mathrm{C}_{23} \mathrm{H}_{21} \mathrm{FN}_{2} \mathrm{O}_{6} \mathrm{~S}: \mathrm{C}, 58.47 ; \mathrm{H}, 4.48 ; \mathrm{N}, 5.93 ; \mathrm{S}, 6.79$. Found: $\mathrm{C}, 58.53 ; \mathrm{H}, 4.51$; N, $5.90 ; \mathrm{S}, 6.62$.

\section{Conclusions}

TDAE methodology is extended here to the reaction of ortho- or para-nitro dichloromethylbenzene derivatives 1-3 with various aromatic $N$-tosylimines $\mathbf{4 a}-\mathbf{g}$, leading to the corresponding aziridines 5-7 in good yields (61-81\%). The diastereoselectivity of the reaction is shown to be sensitive to steric hindrance. Further research is in progress to extent this method to other dichloride derivatives and to explore the ring opening reactions of the aziridines.

\section{Acknowledgments}

This work was supported by the Centre National de la Recherche Scientifique. We express our thanks to V. Remusat for recording the ${ }^{1} \mathrm{H}$ and ${ }^{13} \mathrm{C}-\mathrm{NMR}$ spectra.

\section{Conflict of Interest}

The authors declare no conflict of interest.

\section{References}

1. Müller, P.; Fruit, C. Enantioselective catalytic aziridinations and asymmetric nitrene insertions into ch bonds. Chem. Rev. 2003, 103, 2905-2920.

2. McCoull, W.; Davis, F.A. recent synthetic applications of chiral aziridines. Synthesis 2000, $1347-1365$.

3. Hu, X.E. Nucleophilic ring opening of aziridines. Tetrahedron 2004, 60, 2701-2743.

4. Taylor, A.M.; Schreiber, S.L. Aziridines as intermediates in diversity-oriented syntheses of alkaloids. Tetrahedron Lett. 2009, 50, 3230-3233.

5. $\mathrm{Lu}, \mathrm{P}$. Recent developments in regioselective ring opening of aziridines. Tetrahedron 2010, 66, 2549-2560.

6. Watson, I.D.G.; Yu, L.L.; Yudin, A.K. advances in nitrogen transfer reactions involving aziridines. Acc. Chem. Res. 2006, 39, 194-206. 
7. Davies, H.M.L.; Manning, J.R. Catalytic C-H functionalization by metal carbenoid and nitrenoid insertion. Nature 2008, 451, 417-424.

8. Giri, R.; Shi, B.F.; Engle, K.M.; Maugel, N.; Yu, J.Q. Transition metal-catalyzed C-H activation reactions: diastereoselectivity and enantioselectivity. Chem. Soc. Rev. 2009, 38, 3242-3272.

9. Minakata, S. Utilization of $\mathrm{N}-\mathrm{X}$ bonds in the synthesis of N-Heterocycles. Acc. Chem. Res. 2009, $42,1172-1182$.

10. Collet, F.; Lescot, C.; Dauban, P. Catalytic C-H amination: the stereoselectivity issue. Chem. Soc. Rev. 2011, 40, 1926-1936.

11. Lebel, H.; Spitz, C.; Leogane, O.; Trudel, C.; Parmentier, M. Stereoselective rhodium-catalyzed amination of alkenes. Org. Lett. 2011, 13, 5460-5463.

12. Hansen, K.B.; Finney, N.S.; Jacobsen, E.N. Carbenoid transfer to Imines: A new asymmetric catalytic synthesis of Aziridines. Angew. Chem., Int. Ed. 1995, 34, 676-678.

13. Juhl, K.; Hazell, R.G.; Jørgensen, K.A. Catalytic enantioselective formation of aziridines from $\alpha$ imino esters. J. Chem. Soc., Perkin Trans. 1 1999, 2293-2297.

14. Davis, F.A.; Liu, H.; Zhou, P.; Fang, T.; Reddy, G.V.; Zhang, Y. Aza-Darzens Asymmetric Synthesis of $N$-( $p$-Toluenesulfinyl)aziridine 2-Carboxylate Esters from Sulfinimines ( $N$-Sulfinyl Imines). J. Org. Chem. 1999, 64, 7559-7567.

15. Aggarwal, V.K.; Charmant, J.P.H.; Ciampi, C.; Hornby, J.M.; O'Brien, C.J.; Hynd, G.; Parsons, R. Additions of stabilised and semi-stabilised sulfur ylides to tosyl protected imines: are they under kinetic or thermodynamic control? J. Chem. Soc., Perkin Trans. 1 2001, 3159-3166.

16. Fang, F.; Li, Y.; Tian, S.-K. Stereoselective olefination of N-Sulfonyl Imines with stabilized phosphonium ylides for the synthesis of electron-deficient Alkenes. Eur. J. Org. Chem. 2011, 1084-1091.

17. Takechi, N.; Aït-Mohand, S.; Médebielle, M.; Dolbier, W.R., Jr. Nucleophilic trifluoromethylation of acyl chlorides using the trifluoromethyl iodide/TDAE reagent. Tetrahedron Lett. 2002, 43, 4317-4319.

18. Pooput, C.; Médebielle, M.; Dolbier, W.R., Jr. A new and efficient method for the synthesis of trifluoromethylthio- and selenoethers. Org. Lett. 2004, 6, 301-303.

19. Pooput, C.; Médebielle, M.; Dolbier, W.R., Jr. Nucleophilic perfluoroalkylation of aldehydes, ketones, Imines, Disulfides, and diselenides. J. Org. Chem. 2006, 71, 3564-3568.

20. Montana, M.; Terme, T.; Vanelle, P. Original synthesis of $\alpha$-chloroketones in azaheterocyclic series using TDAE approach. Tetrahedron Lett. 2006, 47, 6573-6576.

21. Montana, M.; Crozet, M.D.; Castera-Ducros, C.; Terme, T.; Vanelle, P. Rapid synthesis of new azaheterocyclic hydroxymalonate derivatives using TDAE approach. Heterocycles 2008, 75, 925-932.

22. Since, M.; Terme, T.; Vanelle, P. Original TDAE strategy using $\alpha$-halocarbonyl derivatives. Tetrahedron 2009, 65, 6128-6134.

23. Juspin, T.; Terme, T.; Vanelle, P. TDAE strategy using $\alpha$-Diketones: Rapid access to 2,3-diphenylquinoline and Acenaphtho[1,2-b]quinoline derivatives. Synlett 2009, 1485-1489.

24. Nadji-Boukrouche, A.R.; Khoumeri, O.; Terme, T.; Liacha, M.; Vanelle, P. Original TDAE reactivity in benzoxa- and benzothiazolone series. ARKIVOC 2010, 358-370. 
25. Montana, M.; Terme, T.; Vanelle, P. TDAE-initiated synthesis of oxiranes in heterocyclic series: Reaction of 2-(Dibromomethyl)quinoxaline with $\alpha$-Dicarbonyl derivatives. Lett. Org. Chem. 2010, 7, 453-456.

26. Juspin, T.; Giuglio-Tonolo, G.; Terme, T.; Vanelle, P. First TDAE-mediated double addition of nitrobenzylic anions to aromatic dialdehydes. Synthesis 2010, 844-848.

27. Khoumeri, O.; Terme, T.; Vanelle, P. Rapid and efficient synthesis of 2-substitutedtetrahydropyrido[3,4-b]quinoxalines using TDAE strategy. Tetrahedron Lett. 2012, 53, 2410-2413.

28. Giuglio-Tonolo, G.; Terme, T.; Médebielle, M.; Vanelle, P. Original reaction of p-nitrobenzyl chloride with aldehydes using tetrakis(dimethylamino)ethylene (TDAE). Tetrahedron Lett. 2003, $44,6433-6435$.

29. Giuglio-Tonolo, G.; Terme, T.; Médebielle, M.; Vanelle, P. Nitrobenzylation of $\alpha$-carbonyl ester derivatives using TDAE approach. Tetrahedron Lett. 2004, 45, 5121-5124.

30. Khoumeri, O.; Terme, T.; Vanelle, P. Original and efficient synthesis of substituted 3,4-Dihydronaphtho[2,3-g]quinoline-2,6,11(1H)-triones. Synthesis 2009, 3677-3683.

31. Khoumeri, O.; Giuglio-Tonolo, G.; Crozet, M.D.; Terme, T.; Vanelle, P. Original synthesis of 2substituted-4,11-dimethoxy-1-(phenylsulfonyl)-2,3-dihydro-1H-naphtho[2,3-f]indole-5,10-diones using TDAE and Cu-catalyzed reaction strategy. Tetrahedron 2011, 67, 6173-6180.

32. Montana, M.; Terme, T.; Vanelle, P. Original synthesis of oxiranes via TDAE methodology: Reaction of 2,2-dibromomethylquinoxaline with aromatic aldehydes. Tetrahedron Lett. 2005, 46, 8373-8376.

33. Khoumeri, O.; Montana, M.; Terme, T.; Vanelle, P. First TDAE approach in quinonic series: Synthesis of new 2-substituted 1,4-dimethoxy-9,10-anthraquinones. Tetrahedron 2008, 64, $11237-11242$.

34. Xu, W.; Dolbier, W.R., Jr. nucleophilic trifluoromethylation of imines using the $\mathrm{cf}_{3} \mathrm{i} /$ tdae reagent. J. Org. Chem. 2005, 70, 4741-4745.

35. Vanelle, P.; De Meo, M.P.; Maldonado, J.; Nouguier, R.; Crozet, M.P.; Laget, M.; Dumenil, G. Genotoxicity in oxazolidine derivatives: Influence of the nitro group. Eur. J. Med. Chem. 1990, $25,241-250$.

36. El-Kashef, H.S.; El-Emary, T.I.; Gasquet, M.; Timon-David, P.; Maldonado, J.; Vanelle, P. New pyrazolo[3,4-b]pyrazines: Synthesis and biological activity. Pharmazie 2000, 55, 572-576.

37. Boufatah, N.; Gellis, A.; Maldonado, J.; Vanelle, P. Efficient microwave-assisted synthesis of new sulfonylbenzimidazole-4,7-diones: Heterocyclic quinones with potential antitumor activity. Tetrahedron 2004, 60, 9131-9137.

38. Gellis, A.; Kovacic, H.; Boufatah, N.; Vanelle, P. Synthesis and cytotoxicity evaluation of some benzimidazole-4,7-diones as bioreductive anticancer agents. Eur. J. Med. Chem. 2008, 43, 1858-1864.

39. Sharghi, H.; Hosseini-Sarvari, M.; Ebrahimpourmoghaddam, S. A novel method for the synthesis of $\mathrm{N}$-sulfonyl aldimines using $\mathrm{AlCl}_{3}$ under solvent-free conditions (SFC). ARKIVOC 2007, 2007, 255-264.

40. Xie, W.; Fang, J.; Li, J.; Wang P.G. Aziridine synthesis in protic media by using lanthanide triflates as catalysts. Tetrahedron 1999, 55, 12929-12938. 
41. Rasmussen, K.G.; Jørgensen, K.A. Catalytic formation of aziridines from imines and diazoacetate. J. Chem. Soc., Chem. Commun. 1995, 1401-1402.

42. Carpenter, W. The Reactions of Tetrakis(dimethylamino)ethylene with Polyhalogenated Compounds. J. Org. Chem. 1965, 30, 3082-3084.

43. Fergus, S.; Eustace, S.J.; Hegarty, A.F. nitrile ylide dimerization: investigation of the carbene reactivity of nitrile ylides. J. Org. Chem. 2004, 69, 4663-4669.

44. Makosza, M.; Owczarczyk, Z. Reactions of organic anions. 161. Dihalomethylation of nitroarenes via vicarious nucleophilic substitution of hydrogen with trihalomethyl carbanions. J. Org. Chem. 1989, 54, 5094-5100.

45. Liu, X.-G.; Wie, Y.; Shi, M. Phosphite-mediated annulation: an efficient protocol for the synthesis of multi-substituted cyclopropanes and aziridines. Tetrahedron 2010, 66, 304-313.

Sample Availability: Samples of the compounds $5 \mathbf{a}-\mathbf{g}, \mathbf{6 a}-\mathbf{g}$ and $7 \mathbf{a}-\mathbf{g}$, are available from the authors.

(C) 2013 by the authors; licensee MDPI, Basel, Switzerland. This article is an open access article distributed under the terms and conditions of the Creative Commons Attribution license (http://creativecommons.org/licenses/by/3.0/). 